\title{
Reconstitution of Giant Mammalian Synapses in Culture for Molecular Functional and Imaging Studies
}

\author{
DDimitar Dimitrov, ${ }^{1}$ Hiroshi Takagi, ${ }^{1}$ Laurent Guillaud, ${ }^{1}$ Naoto Saitoh, ${ }^{2}$ Kohgaku Eguchi, ${ }^{1}$ and Tomoyuki Takahashi ${ }^{1}$ \\ ${ }^{1}$ Cellular and Molecular Synaptic Function Unit, Okinawa Institute of Science and Technology Graduate University, Okinawa, 904-0495, Japan, and \\ ${ }^{2}$ Doshisha University Graduate School of Brain Science, Kyoto 610-0394, Japan
}

Giant presynaptic terminal brain slice preparations have allowed intracellular recording of electrical signals and molecular loading, elucidating cellular and molecular mechanisms underlying neurotransmission and modulation. However, molecular genetic manipulation or optical imaging in these preparations is hampered by factors, such as tissue longevity and background fluorescence. To overcome these difficulties, we developed a giant presynaptic terminal culture preparation, which allows genetic manipulation and enables optical measurements of synaptic vesicle dynamics, simultaneously with presynaptic electrical signal recordings. This giant synapse reconstructed from dissociated mouse brainstem neurons resembles the development of native calyceal giant synapses in several respects. Thus, this novel preparation constitutes a powerful tool for studying molecular mechanisms of neurotransmission, neuromodulation, and neuronal development.

Key words: giant synapse; membrane capacitance measurements; neuronal cell culture; shRNA knockdown; synaptic maturation; vesicle imaging

\section{Significance Statement}

We have developed a novel culture preparation of giant mammalian synapses. These presynaptic terminals make it possible to perform optical imaging simultaneously with presynaptic electrophysiological recording. We demonstrate that this enables one to dissect endocytic and acidification times of synaptic vesicles. In addition, developmental elimination and functional maturation in this cultured preparation provide a useful model for studying presynaptic development. Because this giant synapse preparation allows molecular genetic manipulations, it constitutes a powerful new tool for studying molecular mechanisms of neurotransmission, neuromodulation, and neuronal development.

\section{Introduction}

Central synaptic studies use either acute brain slices or cultures, with their respective technical advantages. Compared with slices, cultured synapses allow genetic manipulations, and their monolayer cell organization makes them more suitable for optical imaging. Furthermore, because of their accessibility for various manipulations, cultured synapses can be used for studying mo-

\footnotetext{
Received 0ct. 26, 2015; revised Feb. 4, 2016; accepted Feb. $22,2016$.

Author contributions: D.D. and T.T. designed research; D.D., H.T., L.G., N.S., and K.E. performed research; D.D., H.T., L.G., N.S., and K.E. analyzed data; D.D. and T.T. wrote the paper.

This work was supported by the Okinawa Institute of Science and Technology and the Core Research for Evolutional Science and Technology of Japan Science and Technology Agency to T.T. We thank Robert Baughman, Takeshi Sakaba, and Shigeo Takamori for comments; Steven D. Aird for editing this manuscript; Toshio Sasaki for assistance with the electron microscopy imaging; Connie Cepko for the gift of pCAG-DsRed plasmid (Addgene \#11151); and Stephen Heinemann and Yongling Zhu for the pcDNA3-SypHluorin 2x plasmid (Addgene \#37004).

The authors declare no competing financial interests.

Correspondence should be addressed to either Dr. Dimitar Dimitrov or Dr. Tomoyuki Takahashi, Cellular and Molecular Synaptic Function Unit, Okinawa Institute of Science and Technology Graduate University, 1919-1, Tancha, 0nna-son, Kunigami, 0kinawa, 904-0495, Japan, E-mail: ddimitrov@oist.jp or ttakahas@oist.jp.

DOI:10.1523/JNEUROSCI.3869-15.2016

Copyright $\odot 2016$ the authors $\quad 0270-6474 / 16 / 363600-11 \$ 15.00 / 0$
}

lecular mechanisms underlying synaptogenesis and synaptic maturation. However, most synapses in culture are too small to access with patch pipettes, and spatially limited for optical imaging. Moreover, the complex input-output relationship often limits detailed analysis of synaptic signals. In acute brain slices, fundamental mechanisms of neurotransmission and neuromodulation have been revealed (Schneggenburger and Forsythe, 2006) using whole-cell patch-clamp recordings from a giant presynaptic terminal, the calyx of Held in auditory brainstem (Forsythe, 1994; Borst et al., 1995; Takahashi et al., 1996). However, because of the tissue thickness and high background fluorescence, slice preparations are generally unsuitable for imaging studies. Moreover, because of limited viability of acute slice preparations, genetic manipulations generally require development of transgenic mice or targeted viral administration in vivo (Wimmer et al., 2006).

To overcome these difficulties, we combined the best attributes of both slice and culture preparations and developed a novel calyx-like giant synapse in culture using dissociated brainstem cells. These synapses share some features with calyces of 
Held synapses in auditory brainstem slices. This culture system also has several technical advantages, enabling us to combine (1) molecular genetic manipulations, (2) high resolution optical imaging in a giant presynaptic terminal structure, and (3) whole-cell recording of electrical signals from presynaptic terminals. Using simultaneous opto-electrical recording, we demonstrate that synaptic vesicle endocytic and vesicle acidification times can be clearly distinguished. Thus, this culture model constitutes a powerful tool for studying molecular mechanisms underlying presynaptic functions and their development.

\section{Materials and Methods}

All experiments were performed in accordance with guidelines of the Physiological Society of Japan and animal experiment regulations at Okinawa Institute of Science and Technology.

Dissociated giant synapse culture. Culture dishes ( $35 \mathrm{~mm} \mu$-dish, Ibidi) or 8 well slides ( $\mu$-slide 8 well, Ibidi) were coated with poly-D-lysine ( $100 \mu \mathrm{g} / \mathrm{ml}$, diluted in $\mathrm{H}_{2} \mathrm{O}$; Millipore) for $1 \mathrm{~h}$ at room temperature, and then washed 3 times with "ultrapure" $\mathrm{H}_{2} \mathrm{O}$ (Millipore). After the wash, the dishes were exposed to air for 2-3 h on a clean bench to dry.

Newborn mice at postnatal day (P) 0 to $\mathrm{P} 1$ (C57BL/6 or ICR strains) of either sex, or embryos (in 18 of 120 culture batches), were taken at gestation day $17-19$ by caesarian section from pregnant mice. Twelve to 50 pups from 1 to 3 litters were used for one round of culture. They were killed by decapitation, and their brains were removed. After removal, a brain was placed on a filter paper soaked in ice-cold HBSS (Invitrogen) with the ventral side facing upward. After removing the meninges, regions containing cochlear nuclei $(\mathrm{CN})$ and the medial nuclei of the trapezoid body (MNTB) were dissected under a stereomicroscope (Leica MZ7.5) using two ophthalmic surgical blades: one for support (\#7635BR; Feather) and the other for cutting (\#K730; Feather). Regions of the CN and MNTB in the superior olivary complex were identified with the aid of a brain atlas (Allen Developing Mouse Brain Atlas; Marrs et al., 2013). For dissecting MNTB region, a $1 \mathrm{~mm}$ coronal incision was made across the brainstem midline on the rostral part of the superior olivary complex to remove pontine nuclei region. Subsequently, another coronal incision was made across the midline on the caudal part of the superior olivary complex $1 \mathrm{~mm}$ away from the first incision. Lateral parts of the superior olivary complex $0.5 \mathrm{~mm}$ away from the midline were then trimmed, connecting the two coronal incisions. A tissue block containing the MNTB region $(1 \mathrm{~mm} \times 1 \mathrm{~mm} \times 0.5 \mathrm{~mm})$ was then excised at $0.5 \mathrm{~mm}$ depth from the ventral surface. Tissue pieces containing CN and MNTB regions were collected separately in different tubes containing ice-cold HBSS, and then dissociated using a papain-based dissociation kit (Nerve Cell Dissociation Medium; Sumitomo Bakelite) following the manufacturer's instructions. The average number of dissociated cells from MNTB and $\mathrm{CN}$ regions were $3.2 \times 10^{4}$ and $1.6 \times 10^{5}$ per pup, respectively (counted using Countess Automated Cell Counter; Invitrogen). In addition to MNTB principal cells or bushy cells, these dissociated cells likely include glia and other neurons. Although we could not specifically label bushy cells or MNTB principal cells, we transfected CN cells with GFP in a majority of cultures (87 of 120) to identify them after coculture (see below). Approximately equal numbers of $\mathrm{CN}$ cells and MNTB cells were taken from each tube, mixed together, and plated at a density of $1.7-2.0 \times 10^{5}$ cells per $35 \mathrm{~mm}$ dish. Cells were cultured in a commercially available serum-free hippocampal astrocyte-conditioned medium (Nerve Cell Culture Medium; Sumitomo) supplemented with nerve growth factor (NGF2.5S, $100 \mathrm{ng} / \mathrm{ml}$, Invitrogen), human brain-derived neurotrophic factor (BDNF, $25 \mathrm{ng} / \mathrm{ml}$, R\&D Systems), human/murine fibroblast growth factor 2 (FGF2, $5 \mathrm{ng} / \mathrm{ml}$, Peprotech), and $\mathrm{KCl}$ concentration was raised to $25 \mathrm{~mm}$. After DIV4, the medium was additionally supplemented with neurotrophin 3 (NT3, $50 \mathrm{ng} / \mathrm{ml}$, Peprotech). The culture medium $(0.8 \mathrm{ml} / 35 \mathrm{~mm}$ dish $)$ was renewed every $4 \mathrm{~d}$ by exchanging half its volume. Cytosine arabinoside $(5 \mu \mathrm{M})$ was used to stop glial cell proliferation at DIV8. For NT3 neutralization, $8-10 \mu \mathrm{g} / \mathrm{ml}$ anti-NT3 antibody (500-P82G; Peprotech), or normal goat IgG (sc-2028; Santa Cruz Biotechnology) for controls, was added during medium change from DIV4 onward.
Complementary DNA cloning, transfections, and shRNA knockdown. For labeling $\mathrm{CN}$ neurons and giant presynaptic terminals, cytosolic AcGFP1 (Takara) was overexpressed in cells. To prepare the AcGFP1 cDNA construct, the AcGFP1 coding sequence (pAcGFP1; Takara) was cloned into the pCAG-DsRed (Matsuda and Cepko, 2004) vector in the place of DsRed. For imaging of exocytosis/endocytosis in the cultured neurons, synaptophysin (syp)-pHluorin fusion protein was overexpressed in calyceal terminals and imaged after field stimulation with a bipolar electrode. To prepare the pHluorin construct, sypHluorin $2 \mathrm{x}$ was extracted from pcDNA3-sypHluorin 2x (a gift from Stephen Heinemann and Yongling Zhu; Addgene plasmid \# 37004) (Zhu et al., 2009) and inserted into the pCAG-DsRed vector in the place of DsRed.

Transfections were performed with the Neon transfection system (Invitrogen). Immediately before plating, dissociated CN cells were suspended in electroporation buffer (Neon transfection system) at a density of $0.8-1.0 \times 10^{7} / \mathrm{ml}$ and electroporated $(1250-1350 \mathrm{~V}, 20-23 \mu \mathrm{s}, 1$ pulse) in the presence of $0.8 \mu \mathrm{g}$ cDNA per $10 \mu \mathrm{l}$ cell suspension. After electroporation, the number of cells was adjusted and plated as described above. In addition to the manufacturer's protocol, we kept the cells with the cDNA on ice for 10 min before electroporation. The efficiency of GFP transfection was consistently $\sim 20 \%$, which was estimated in $\mathrm{CN}$ alone cultures by counting the number of GFP-labeled cells out of total CN cells observed with DIC optics.

Lentiviral-mediated shRNA knockdown of VGLUT2 was accomplished with commercially available lentiviral particles (sc-42333-V; Santa Cruz Biotechnology); $3.3 \times 10^{5}$ units of lentiviral particles was added to one $35 \mathrm{~mm}$ dish of culture at DIV8. Cultures were then processed normally and examined at DIV18. For controls, cultured cells were infected with control lentiviral particles (sc-108080; Santa Cruz Biotechnology). Knockdown efficiency was estimated by comparing the fluorescence intensity (integrated density) of VGLUT1- and VGLUT2immunostained calyceal terminals.

Immunofluorescence. Cultured cells were fixed with PFA (4\% in PBS) for $15 \mathrm{~min}$ at $37^{\circ} \mathrm{C}$, permeabilized with Triton X-100 (0.1\%, Nacalai Tesque) for $5 \mathrm{~min}$ at room temperature $\left(26^{\circ} \mathrm{C}-27^{\circ} \mathrm{C}\right)$, and blocked in normal goat serum (10\% v/v; Vector Laboratories) for 30-60 min at room temperature. Fixed samples were incubated with primary antibodies for $2 \mathrm{~h}$ at room temperature, or overnight at $4^{\circ} \mathrm{C}$ on a shaker. After incubation, samples were washed and incubated with secondary antibodies (1:200 diluted in PBS) for $2 \mathrm{~h}$ at room temperature, and imaged in PBS. The primary antibodies were anti-VGLUT1 guinea pig antiserum (1:5000, AB5905; Millipore), anti-VGLUT2 mouse monoclonal clone 8G9.2 (1:1000, ab79157; Abcam), and mouse anti-PSD95 (1:200, catalog \#124001; Synaptic Systems). The secondary antibodies were goat IgG conjugated with AlexaFluor-405, -488, or -568 (Invitrogen).

Surface receptor labeling. Surface GluR subunit labeling was performed as previously described (Allen et al., 2012). Antibodies against the GluR subunit N-terminal domains (anti-GluR1-NT clone RH95; and antiGluR2 clone 6C4; Millipore) were added directly to the live culture at a final concentration of $10 \mu \mathrm{g} / \mathrm{ml}$ for $15 \mathrm{~min}$ in a culture incubator $\left(37^{\circ} \mathrm{C}\right.$, $5 \% \mathrm{CO}_{2}$ ). After incubation, cells were washed with PBS, fixed with PFA ( $4 \%$ in PBS) for $15 \mathrm{~min}$ at $37^{\circ} \mathrm{C}$, and processed for immunofluorescence examination as described above.

Patch-clamp recording of postsynaptic currents. For measurements of postsynaptic currents, whole-cell voltage-clamp recordings were made from cells that were visually determined to be in contact with a GFPexpressing giant calyceal terminal. Whole-cell recordings were performed with patch pipettes pulled from borosilicate glass capillary tubes (GC150TF-10; Warner Instruments) using a pipette puller (model P-97; Sutter Instruments). The whole-cell pipette solution for postsynaptic recording contained $(\mathrm{mM})$ as follows: $30 \mathrm{KCl}, 105 \mathrm{~K}$-gluconate, 10 HEPES, $12 \mathrm{Na}_{2}$-phosphocreatine, $1 \mathrm{~L}$-arginine, $1 \mathrm{MgCl}_{2}$, $3 \mathrm{MgATP}, 0.5$ EGTA, pH 7.3, adjusted with $\mathrm{KOH}$. The recording chamber was perfused with standard aCSF (in $\mathrm{mM}$ ) as follows: $125 \mathrm{NaCl}, 2.5 \mathrm{KCl}, 2 \mathrm{CaCl}_{2}, 1$ $\mathrm{MgCl}_{2}, 10$ glucose, $1.25 \mathrm{NaH}_{2} \mathrm{PO}_{4}, 2$ Na-pyruvate, 3 myo-inositol, 0.5 ascorbic acid, $26 \mathrm{NaHCO}_{3}$, pH 7.3 aerated with $\mathrm{O}_{2} / \mathrm{CO}_{2}$ (95/5\%), and kept at $37^{\circ} \mathrm{C}$ with a TC344B dual temperature controller coupled to an SH27B inline heater (Warner Instruments). Postsynaptic pipette resistance was 3-7 $\mathrm{M} \Omega$, and access resistance $(8-10 \mathrm{M} \Omega$ ) was not compen- 
sated. Synaptic currents were recorded with an Axopatch 200B amplifier (Molecular Devices). EPSCs were evoked by stimulation (10-40 V, 150$900 \mu$ s, using a SEN-8200 stimulator, Nihon Kohden) with a bipolar electrode (FHC) placed near GFP-expressing neurites extending to calyceal terminals on postsynaptic neurons. Electrical stimulation was synchronized with Clampex 10 data acquisition software (Molecular Devices). Standard aCSF contained $10 \mu \mathrm{m}$ bicuculline methiodide and 0.5 $\mu \mathrm{M}$ strychnine hydrochloride. For miniature EPSC (mEPSC) recordings, the bath solution also contained $0.4 \mu \mathrm{M} \mathrm{TTX}$, and the $\mathrm{KCl}$ concentration in aCSF was raised from 2.5 to $25 \mathrm{~mm}$ ( $\mathrm{NaCl}$ was reduced by $22.5 \mathrm{~mm}$ ) to increase $\mathrm{mEPSC}$ frequency.

Patch-clamp recording from calyceal presynaptic terminals. Presynaptic terminals of cultured calyceal terminals (DIV18-DIV20) were identified with a $40 \times$ water-immersion objective attached to an upright microscope (BX51WI; Olympus). Presynaptic pipette internal solution contained (mM) the following: 125 Cs-methanesulfonate, $30 \mathrm{CsCl}, 10$ HEPES, 0.5 EGTA, $12 \mathrm{Na}_{2}$-phosphocreatine, $3 \mathrm{MgATP}, 1 \mathrm{MgCl}_{2}, 0.3$ $\mathrm{Na}_{2}$ GTP (315-320 mOsm, pH 7.3 adjusted with $\mathrm{CsOH}$ ). To label patched terminals after whole-cell recording, AlexaFluor-594 $(50 \mu \mathrm{M})$ was included in the pipette solution. Resistances of patch electrodes for presynaptic recording were $10-15 \mathrm{M} \Omega$, and series resistances were 15-30 $\mathrm{M} \Omega$, which was compensated by up to $70 \%$ for a final value of $10 \mathrm{M} \Omega$. After establishing whole-cell recording, membrane capacitance measurements were performed as previously described (Eguchi et al., 2012). Briefly, calyceal terminals were voltage-clamped at a holding potential of $-80 \mathrm{mV}$, and a sinusoidal voltage command with a peak-to-peak voltage of $60 \mathrm{mV}$ was applied at $1 \mathrm{kHz}$. To isolate presynaptic voltagegated $\mathrm{Ca}^{2+}$ currents $\left(\mathrm{I}_{\mathrm{Ca}}\right.$ ), the aCSF contained $10 \mathrm{~mm}$ tetraethylammonium chloride, $0.5 \mathrm{~mm}$ 4-aminopyridine, $1 \mu \mathrm{M}$ TTX, $10 \mu \mathrm{M}$ bicuculline methiodide, and $0.5 \mu \mathrm{M}$ strychnine hydrochloride. Recording pipette tips were coated with dental wax to minimize stray capacitance $(4-6 \mathrm{pF})$. Single square pulse (duration, $10 \mathrm{~ms}$ ) or a $20 \mathrm{~Hz}$ train of depolarizing pulses (20 ms to $10 \mathrm{mV}, 20$ times) was used to induce presynaptic $\mathrm{I}_{\mathrm{Ca}}$. Membrane capacitance changes $\left(\mathrm{C}_{\mathrm{m}}\right)$ within $450 \mathrm{~ms}$ of stimulation were excluded from analysis to avoid contamination of conductancedependent capacitance artifacts. Sample records of $C_{m}$ are shown as average values of each 50 data points (for $50 \mathrm{~ms}$ ) plotted every $0.5 \mathrm{~s}$. Data were acquired at a sampling rate of $50 \mathrm{kHz}$, using an EPC-10 patch-clamp amplifier controlled by PatchMaster software (HEKA Elektronik) after online filtering at $5 \mathrm{kHz}$. Experiments were performed at $37^{\circ} \mathrm{C}$.

Transmission electron microscopy. Standard transmission electron microscopy protocol was used. Briefly, samples were fixed in 4\% PFA and $1 \%$ glutaraldehyde in $0.1 \mathrm{M}$ phosphate buffer overnight and postfixed with $1 \% \mathrm{OsO}_{4}$ in $0.1 \mathrm{~m}$ phosphate buffer for $1 \mathrm{~h}$. After successive dehydration in ethanol, samples were embedded in Epon 812 and processed for sectioning on an ultramicrotome (UC6, Leica Microsystems). Ultrathin sections $(70 \mathrm{~nm})$ were stained with uranyl acetate and lead citrate for $15 \mathrm{~min}$ and observed on a transmission electron microscope (JEM-1230R, JEOL) at $100 \mathrm{KeV}$.

Live imaging of synaptic vesicle dynamics. FM 4-64 dye labeling and imaging were performed as previously reported (Gaffield and Betz, 2006). The culture dish was continuously perfused with standard aCSF containing $25 \mu \mathrm{M} C N Q X$. In high $\left[\mathrm{K}^{+}\right]$aCSF solution $(80 \mathrm{~mm} \mathrm{KCl})$, $\mathrm{NaCl}$ was replaced by KCl. FM 4-64 (Invitrogen; or SynapseRed C2, Promokine) was used at a final concentration of 2.0-3.0 $\mu \mathrm{M}$.

FM dye was loaded into presynaptic terminals by incubating it in standard aCSF, and then in high $\left[\mathrm{K}^{+}\right] \mathrm{aCSF}$, followed by an 8-10 min washout with standard aCSF containing the dye. Before imaging extracellular FM dye was washed out for 12-15 min.

For pHluorin imaging, $\mathrm{CN}$ neurons in calyceal cultures were transfected with pCAG-sypHluorin $2 x$ during culture plating. On the day of experiments, a culture dish was set on the imaging stage and perfused with aCSF. Calyceal terminals were identified using resting pHluorin fluorescence. Time-lapse imaging was performed over a portion or over a whole calyceal terminal at 1-3 frames per second simultaneously with patch-clamp recording. Simultaneous imaging of pHluorin with capacitance measurements was performed using an EMCCD camera (ImagEM) controlled by Aquacosmos software (Hamamatsu Photonics) at 2 frames per second.
For vesicle imaging, CypHer5E dye was loaded into synaptic vesicles in GFP-labeled presynaptic terminals in culture. One day before experiments, cultures were incubated overnight with CypHer5E dye conjugated to synaptotagmin 2 luminal domain antibodies $(105223 \mathrm{CpH}$; Synaptic Systems). The next day, culture dishes were perfused and imaged. Bath solutions were perfused using a peristaltic pump with aCSF controlled at $37^{\circ} \mathrm{C}$.

Image acquisition. Standard confocal images were acquired on an LSM710 confocal microscope (Carl Zeiss) with a $40 \times$ objective lens (C-Apochromat, NA 1.2, Carl Zeiss) for live FM dye or pHluorin imaging and electrophysiological recordings, or a $63 \times$ objective (PlanApochromat, NA 1.4 Oil DIC M27, Carl Zeiss) for fixed sample imaging. Live imaging of CypHer5E loaded in terminals was done with a $100 \times$ objective ( $\alpha$ Plan-Fluar, 1.45 NA, Oil, Carl Zeiss). Long-term live cell monitoring was performed using a Biostation IM-Q incubating microscope (Nikon); fluorescence images $(1280 \times 960,14$ bit $)$ were acquired with a standard GFP filter set (excitation at $470 \mathrm{~nm}$, emission at $523 \mathrm{~nm}$ ) and a $40 \times$ objective ( $0.8 \mathrm{NA}$ Plan Fluor). Images at $8 z$-axis planes $(2 \mu \mathrm{m}$ $z$-steps, $14 \mu \mathrm{m}$ in total $z$-range) were obtained for each time point (every $2 \mathrm{~h}$ ) and assembled.

Data and statistical analyses. Images were processed using ZEN 2010 (Carl Zeiss), ImageJ (National Institutes of Health), and Adobe Illustrator CS5 (Adobe) software packages. For FM dye and pHluorin imaging, to quantify the absolute change of the fluorescence signal $(\Delta \mathrm{F} / \mathrm{F})$, the average fluorescence intensity $(\mathrm{F})$ of a region of interest was quantified (ImageJ), corrected for photo-bleaching, and corrected by the baseline fluorescence intensity $\left(\mathrm{F}_{0}\right)$ as $\left(\mathrm{F}-\mathrm{F}_{0}\right) / \mathrm{F}_{0}$ at each time point. Correction for photo-bleaching was made by subtracting a trace calculated from a double or single exponential decay function (best fit) of traces before and after stimulation. Half-decay time was measured as the time required for the signal to reach half of its peak intensity. Signal-to-noise ratio was calculated as the ratio between the mean peak fluorescence intensity and the SD of the baseline fluorescence intensity. Images from the Biostaion IM-Q were additionally processed using the "AutoQuant" blind deconvolution module in the NIS-Elements AR software package (Nikon). CypHer5E trajectory tracing was done with the Imaris 7.1.1 software package (Bitplane). Electrophysiological data were analyzed using the Clampfit 10.2 software package (Molecular Devices). Miniature EPSC analysis was performed using the Mini Analysis Program 6.07 software package (Synaptosoft). Data analysis and graphing were performed using the OriginPro 8.6 software package (OriginLab) or IgorPro (Wave Metrics) for Figure 4. In bar graphs, values and numbers are presented as mean \pm SEM. For statistical analysis, one-way ANOVA with the TukeyKramer post hoc test was used unless otherwise noted. Statistical significance was set at $p<0.05$.

\section{Results}

\section{Formation of calyceal giant synapses in dissociated brainstem cell coculture}

In mammalian brainstem, globular bushy cells from the anterior ventral cochlear nucleus extend their axons to form giant calyx of Held synapses with principal neurons in the contralateral MNTB by postnatal days (P) 2 to P4 (Hoffpauir et al., 2006). These synapses are named "calyx" because of their large presynaptic structures wrapping postsynaptic cells (Ramon y Cajal, 1911). Calyceal synapses are also formed on vestibular hair cells (Wellsall, 1956) and in chick ciliary ganglia (De Lorenzo, 1960). To realize formation of calyceal synapses in culture, we excised the $\mathrm{CN}$ - and MNTB-containing regions from newborn or fetal mouse brains and dissociated them enzymatically. To identify giant axon terminals of $\mathrm{CN}$ neurons in culture, we labeled CN cells with GFP by transfecting a cytosolic GFP expression vector, before coculture. During coculture in astrocyte preconditioned media, GFP-labeled CN cells extended their neurites and formed contacts with GFP-negative large neurons, possibly MNTB neurons (Fig. 1a).

Calyceal synaptic contacts were occasionally seen after a week in culture but were constantly observed at approximately the 
a
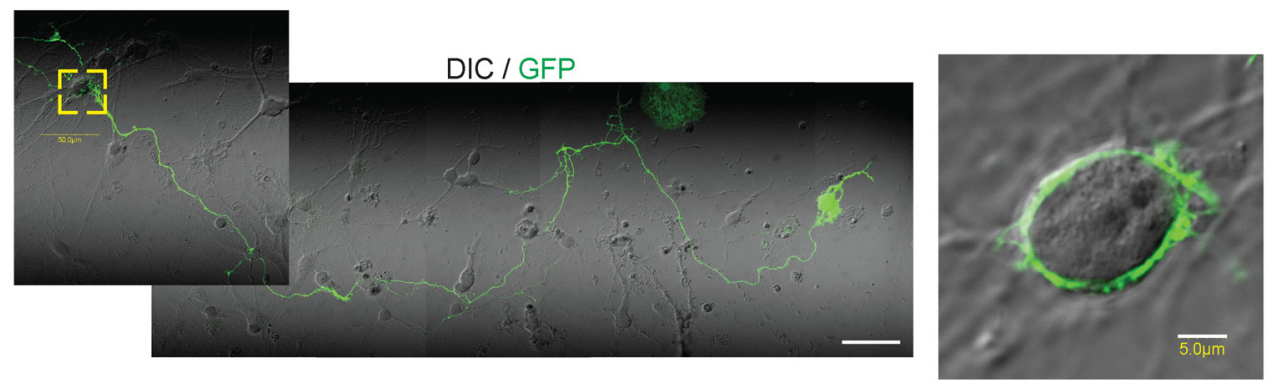

b
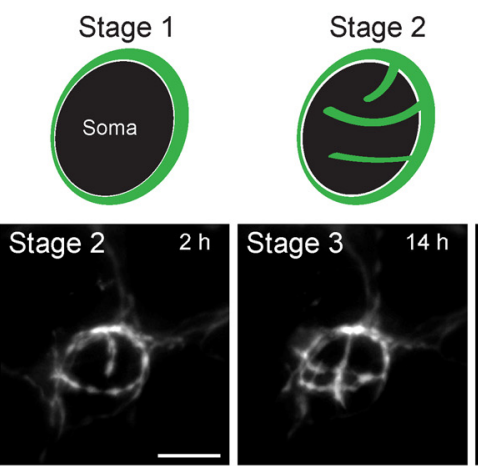
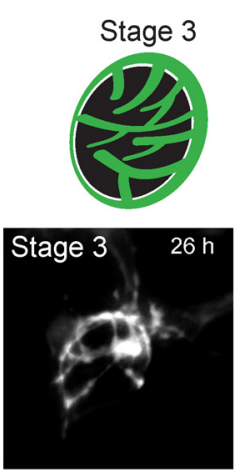

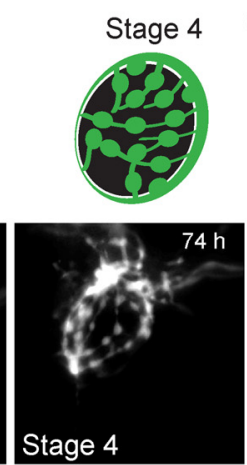

C

d
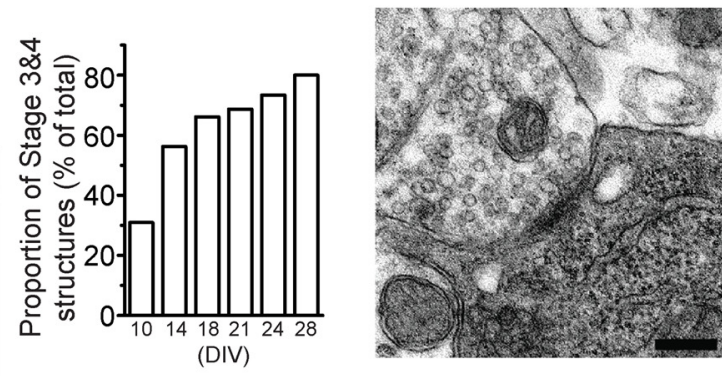

e
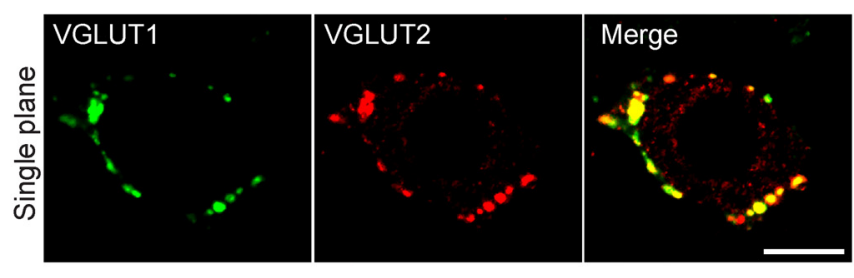

9
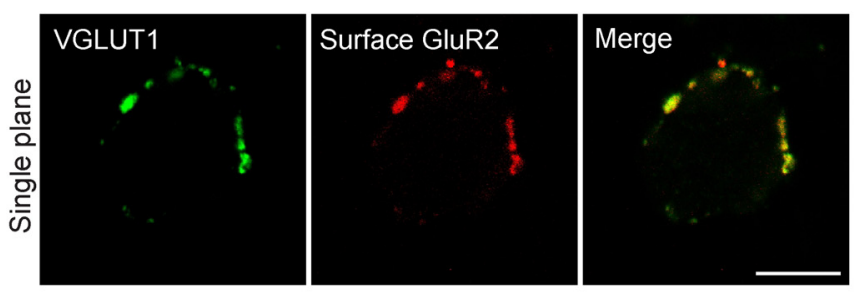

h

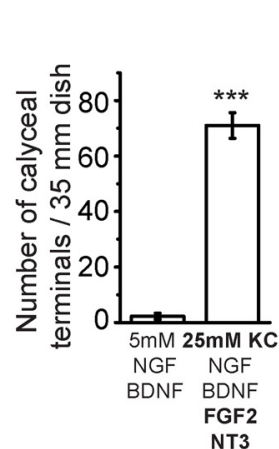

i

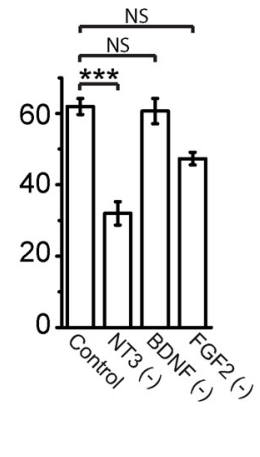

j

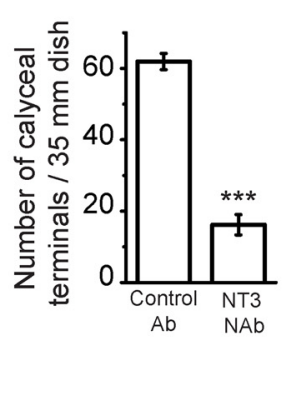

f Max projection

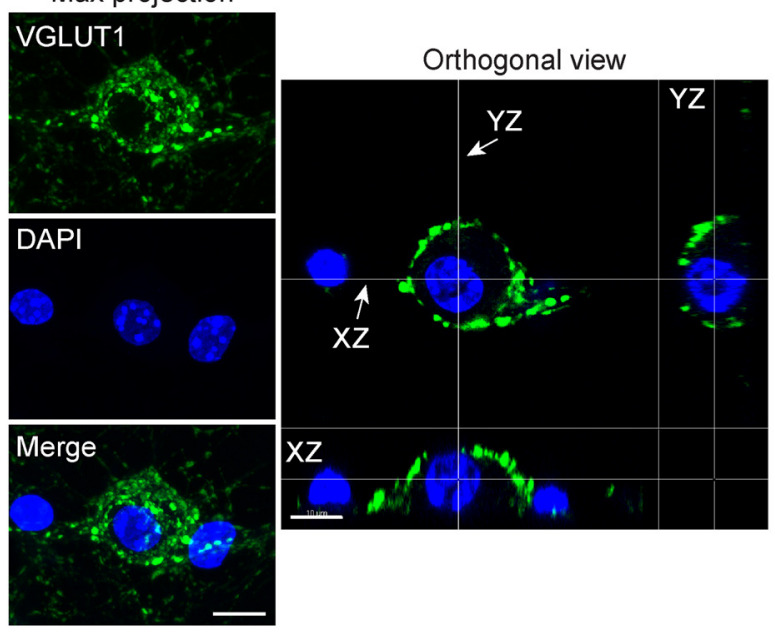

k

Figure 1. Formation of calyceal giant synapses in a dissociated cell culture system. $\boldsymbol{a}$, A giant calyceal terminal in culture, visualized with GFP overexpressed in cells derived from neonatal mice cochlear nuclei. Left, Merged DIC and GFP fluorescent images represent a GFP-transfected neuron with its axon contacting a nontransfected neuron (yellow box). Scale bar, 50 mm. Right, Upper confocal plane zoom-in view of the calyceal contact in the box. Scale bar, $5 \mu \mathrm{m}$. $\boldsymbol{b}$, Structural classification of GFP-labeled calyceal terminals into 4 stages. Live fluorescence imaging of a GFP-expressing calyceal terminal, for a total span of $74 \mathrm{~h}$, starting at DIV13 (Movie 1). Scale bar, $10 \mu \mathrm{m}$. c, Proportion of late-stage (Stages 3 and 4 ) calyces at different DIVs (10 $-28, n=3$ dishes at each DIV). $\boldsymbol{d}$, Transmission electron microscope image of a cultured calyceal terminal swelling in contact with a postsynaptic cell, showing clear vesicles and a mitochondrion (DIV14). Scale bar, $200 \mathrm{~nm} . \boldsymbol{e}$, VGLUT1 (green) and VGLUT2 (red) immunofluorescence of a single calyceal terminal shown at a single confocal plane. Scale bar, $10 \mu \mathrm{m}$. $\boldsymbol{f}$, Immunofluorescence confocal $z$-stack maximum projections of a giant terminal stained for VGLUT1 (green) and the nuclear marker DAPI (blue), merged image and orthogonal view. Scale bar, $10 \mu \mathrm{m} . \boldsymbol{g}$, VGLUT1 (green) and surface GluR2 (red) immunofluorescence of a single calyceal terminal shown at a single confocal plane. Scale bar, $10 \mu \mathrm{m}$. $\boldsymbol{h}$, The number of FM dye-stained calyces in a $35 \mathrm{~mm}$ culture dish. Left bar, In astrocyte-

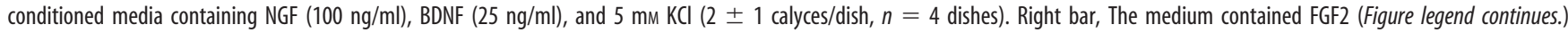


10th day in vitro (DIV10). The number of GFP-labeled calyceal terminals increased steeply thereafter. Just after contact, terminals appeared as simple ring-like structures (Fig. 1b). During the next $3 \mathrm{~d}$, they underwent marked structural remodeling (Fig. $1 b$; Movie 1). Ring-like terminals grew upward and extended their finger-like processes over the surfaces of postsynaptic neurons (Fig. $1 b$ ). Subsequently, the number of fingers increased and secondary fingers emerged. Finally, swellings appeared along the fingers. For practical purpose, we classified calyceal terminals developing in culture into four stages: Stage 1 displayed ring-like structures; Stage 2 displayed finger-like structures; Stage 3 displayed secondary fingers; and Stage 4 displayed swellings. Throughout DIV10-DIV28, calyceal structures of different stages coexisted, with the proportion of mature structures (Stage 3 and 4) increasing from DIV10 to DIV28 (Fig. 1c). In transmission electron micrographs, synaptic vesicles were observed together with mitochondria in the swellings of cultured calyceal terminals at Stage 4 (Fig. 1d).

Calyceal presynaptic terminals expressed vesicular transporters VGLUT1 and VGLUT2 (Fig. 1e), indicating that they are glutamatergic presynaptic terminals. VGLUT1 and VGLUT2 immunofluorescence overlapped only partially as at calyx of Held terminals in brainstem slices (L.G. et al., unpublished observation). VGLUT1-labeled calyceal terminals wrapped the whole postsynaptic cell body, the nucleus of which was stained with DAPI (Fig. 1f). Postsynaptic cells expressed GluR2 on their surfaces (Fig. 1g) and GluR1 (data not shown), labeled with their respective extracellular epitope antibodies. Postsynaptic surface GluR2 and presynaptic VGLUT1 immunofluorescence showed a large overlap, suggesting that postsynaptic AMPA receptors are expressed in apposition to presynaptic terminals.

To induce formation of calyceal giant synapses, it was necessary to increase $\mathrm{KCl}$ concentration (from 5 to $25 \mathrm{~mm}$ ) in culture media (Lohmann et al., 1998; Tong et al., 2010) and to add FGF2 (5-10 ng/ml) and NT3 (50 ng/ml). Under these culture conditions, the number of giant calyceal synapses, visualized with FM-

(Figure legend continued.) $\quad(5 \mathrm{ng} / \mathrm{ml}), \mathrm{NT3}(50 \mathrm{ng} / \mathrm{ml})$, and $25 \mathrm{~mm} \mathrm{KCl}(71 \pm 5$ calyces/dish, $n=3$ dishes from three independent cultures, DIV13). ${ }^{* * *} p<0.001$. NS, Not significant. $\boldsymbol{i}$, Numbers of FM dye-labeled calyceal terminals per $35 \mathrm{~mm}$ dish in control culture media (62 \pm 2 calyces, $n=6$ dishes), after omission of NT3 ( $32 \pm 3$ calyces, $n=5$ dishes), BDNF (61 \pm 4 calyces, $n=5$ dishes), or FGF2 ( $47 \pm 2$ calyces, $n=5$ dishes) from the culture media. $j$, The number of FM dye-labeled calyceal terminals per $35 \mathrm{~mm}$ culture dish, treated with normal lgG (Control) or NT3-neutralizing antibody (NT3 NAb, $8-10 \mu \mathrm{g} / \mathrm{ml})$. The numbers are $63 \pm 3(n=$ 14 dishes) in control and $16 \pm 3(n=10, p<0.001)$ in NT3 NAb-treated dishes. $\boldsymbol{k}$, Small synaptic puncta on neurites identified with VGLUT1 (green) and PSD95 (red) double immunofluorescence staining, in cultures treated with control antibody (top) or NT3 NAb (bottom). Bar graphs represent the number of VGLUT1- and PSD95-positive presynaptic puncta per $50 \mu \mathrm{m}$ length of neurites in control $(12.7 \pm 0.45, n=32)$ and NT3 NAb-treated cultures (13.0 \pm 0.47 , $n=34)$. Experiments were performed at DIV14-DIV18. Scale bar, $10 \mu \mathrm{m}$. dye loading, was $\sim 70$ per $35 \mathrm{~mm}$ dish, whereas without these supplements there were $<3$ giant synapses per dish (Fig. $1 h$ ). In a separate set of experiments, the average number of GFP-positive presynaptic terminals was 13 per dish in CN-MNTB coculture, whereas it was 2 in $\mathrm{CN}$ alone cultures. These numbers are as expected from the efficiency of GFP transfection in CN cells (20\%; see Materials and Methods). NT3 contributed significantly to calyceal synapse formation because its omission from culture media reduced FM-dye labeled calyceal synapses to $52 \%$ (Fig. 1i). In contrast, omission of BDNF from culture media had no effect on calyceal synapse formation (Fig. 1i). Removal of NGF from culture media resulted in unhealthy neurons that die within the first week of culture (data not shown). Removal of FGF2 tended to reduce the number of calyceal synapses formed (Fig. $1 i ; p=0.10$ ). To evaluate the contribution of endogenous NT3 present in the conditioned medium, we omitted NT3 from the supplement mixture and added an NT3-neutralizing antibody. This antibody treatment reduced FM dye-labeled calyceal synaptic formation to $26 \%$ (Fig. 1 j), more strongly than the mere omission of NT3 from culture media (56\%). To investigate whether NT3 is specifically required for calyceal synaptic formation, we tested the effect of NT3 antibody on small glutamatergic synaptic puncta that coexpress VGLUT1 and PSD95. Under DIC optics, we selected puncta on fine neurites remote from cell bodies to exclude swellings of calyceal terminals. These puncta show VGLUT1 and PSD95 immunofluorescence coexisting in apposition (Fig. 1k). NT3 antibody had no significant effect on the number of such small puncta. Thus, in CN-MNTB coculture, NT3 specifically promotes calyceal giant synapse formation without affecting conventional synapse formation. 
a

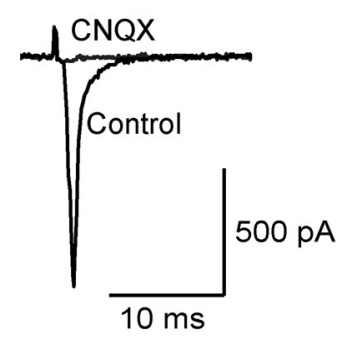

C

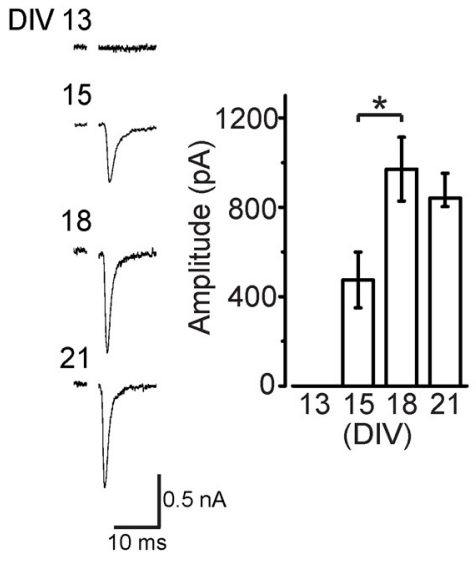

b

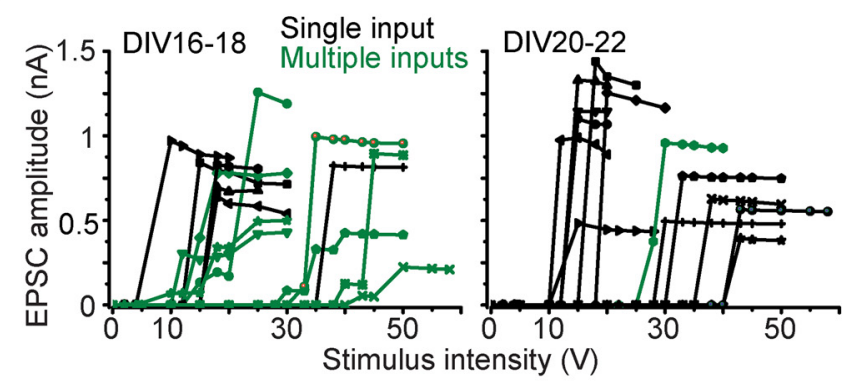

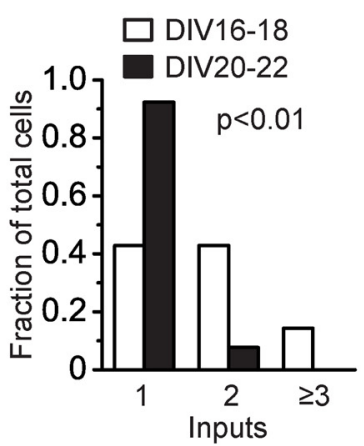

d
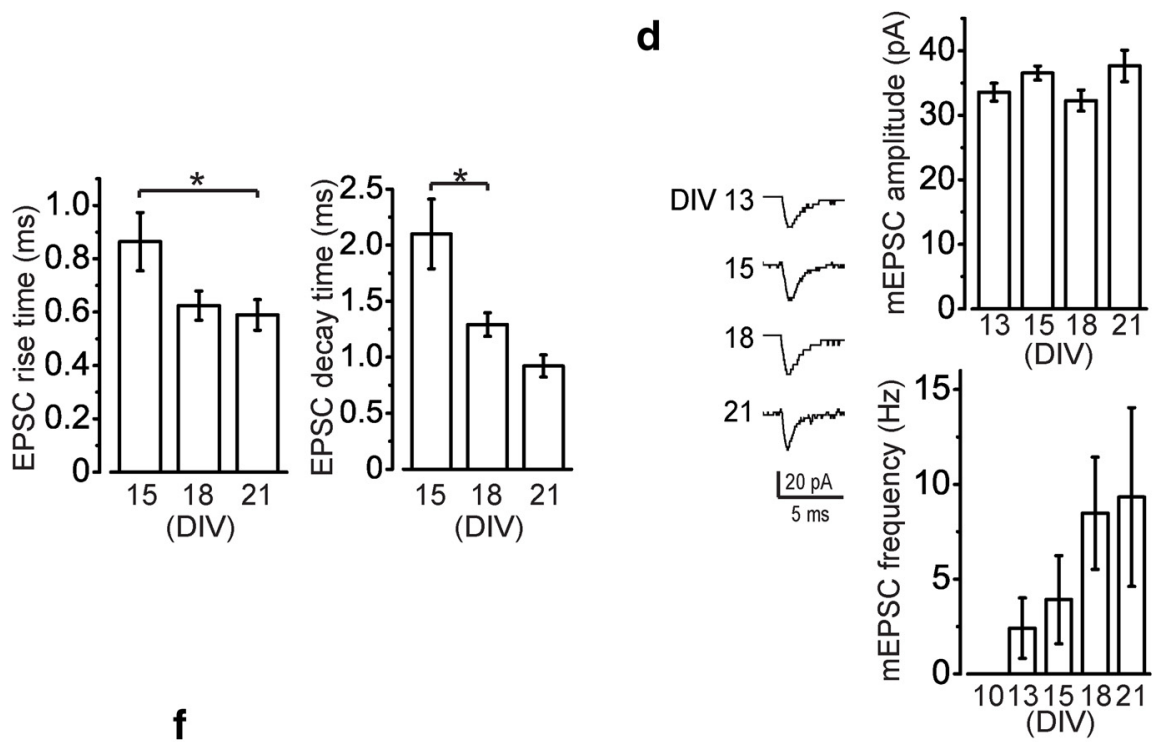

e

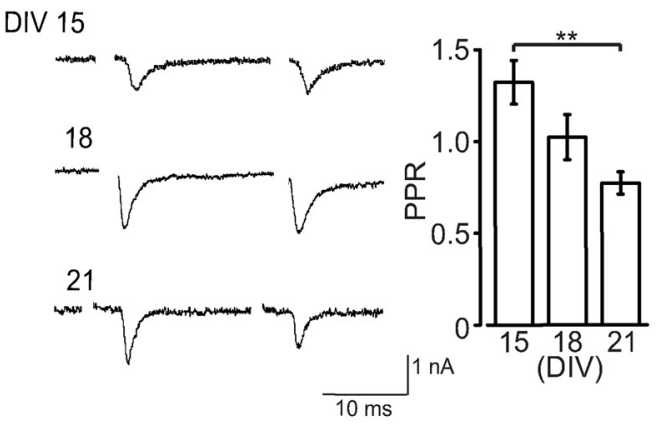

f

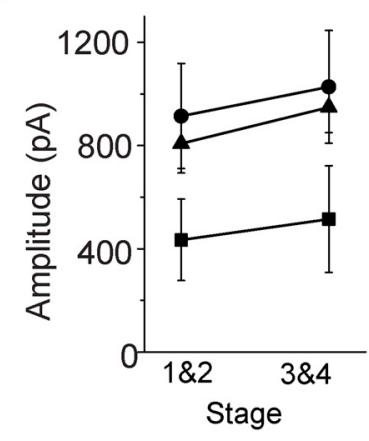

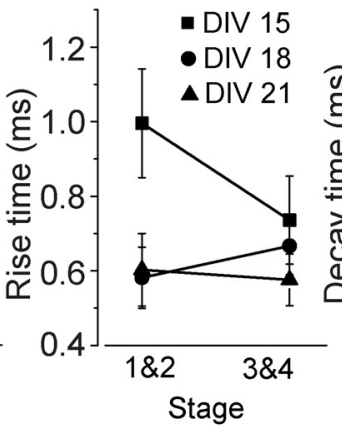

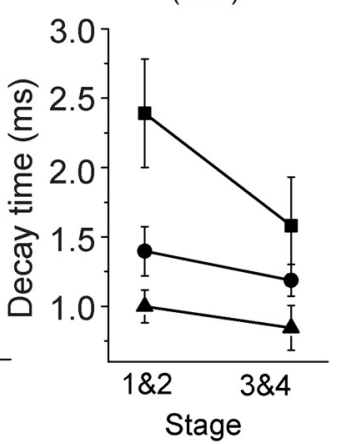

Figure 2. Functional maturation of calyceal giant synapses in culture. $\boldsymbol{a}$, EPSCS evoked by nerve fiber stimulation in a cell contacted by a GFP-labeled calyceal terminal (DIV15). EPSCS were abolished by bath application of CNQX ( $25 \mu \mathrm{M}$, superimposed). $\boldsymbol{b}$, Amplitudes of EPSCs evoked in response to gradually increased stimulus intensity at GFP-labeled calyceal synapses in DIV16-DIV18 and DIV20-DIV22. EPSCs were evoked either in multiple steps (green) or in a single step (black). Bar graphs represent the number of inputs (1,2, and $>3$ ) at DIV16-DIV18 (open bars) and DIV 20-DIV22 (filled bars), which are statistically significant (Mann-Whitney test, $p<0.01$ ). c, Averaged EPSCs (from all events) evoked by nerve fiber stimulation at different DIVs. No EPSCs could be evoked at DIV13 $(n=9)$. EPSCs were evoked after DIV 15, increased in amplitude from DIV15 to DIV18 $\left({ }^{*} p<0.05\right)$, and remained constant thereafter (left bar graphs). Middle and right bar graphs represent a 10\%-90\% rise time and decay time constant. Rise time constant was $0.86 \pm 0.1,0.62 \pm 0.06$, and $0.59 \pm 0.06 \mathrm{~ms}$, and decay time constant was $2.1 \pm 0.3,1.29 \pm 0.11$, and $0.92 \pm 0.1 \mathrm{~ms}$ at DIV15, DIV18, and DIV21, respectively. ${ }^{*} p<0.05 .{ }^{* *} p<0.01 . \boldsymbol{d}$, mEPSCs recorded in high [K $\left.{ }^{+}\right](25 \mathrm{~mm})$ aCSF at DIV13-21. At DIV10, $\mathrm{mEPSC}$ were detectable in only 1 of 5 cells (data not shown). Sample records of mEPSCs were averaged from 50 to 200 events. Mean mEPSC amplitudes were $34 \pm$ $1.4,37 \pm 1.1,32 \pm 1.6$, and $38 \pm 2.5 \mathrm{pA}$ at DIV13, DIV15, DIV18, and DIV21, respectively $(p=0.15)$. Mean frequencies of $\mathrm{mEPSC}$ were $2.4 \pm 1.6,3.9 \pm 2.3,8.5 \pm 3.0$, and $9.3 \pm$ $4.7 \mathrm{~Hz}$ at DIV13, DIV15, DIV18, and DIV21, respectively ( $n=4$ cells each, $p=0.36$ ). e, EPSCs evoked by a paired-pulse protocol at DIV15, DIV18, and DIV21(interpulse interval, $20 \mathrm{ms,}$ stimulation artifacts are truncated). Bar graphs represent PPR (the second amplitude relative to the first) of evoked EPSCs at DIV15 $(1.32 \pm 0.12, n=14)$, DIV18 (1.02 $\pm 0.12, n=12$ ), and DIV21 $(0.77 \pm 0.06, n=14, p=0.002)$. $f$, Lack of structural and functional correlation in developing calyceal synapses in culture. Average EPSC amplitude and kinetics in cultured calyceal synapses at structural Stages 1 and 2 or Stages 3 and 4 at DIV15, DIV18, and DIV21. At DIV15, EPSC amplitudes at Stages 1 and 2 and Stages 3 and 4 were $434 \pm 158(n=6)$ and $515 \pm 207 \mathrm{pA}(n=6)$, respectively (no significant difference, $p=0.76)$. EPSC rise times $(10 \%-90 \%)$ were $0.99 \pm 0.15$ and $0.74 \pm 0.12 \mathrm{~ms}$, respectively $(p=0.19)$, EPSC decay times were $2.39 \pm 0.39$ and $1.58 \pm 0.35 \mathrm{~ms}$, respectively $(p=0.15)$. At DIV18, EPSC amplitudes at Stages 1 and 2 and Stages 3 and 4 were $914 \pm 203(n=6)$ and $1027 \pm 218 \mathrm{pA}(n=6)$, respectively $(p=0.71)$, rise times were $0.58 \pm 0.08$ and $0.67 \pm 0.08 \mathrm{~ms}$, respectively $(p=0.47)$, and decay times were $1.4 \pm 0.18$ and $1.19 \pm 0.11 \mathrm{~ms}$, respectively $(p=0.34)$. At DIV21, EPSC amplitudes at Stages 1 and 2 and Stages 3 and 4 were $807 \pm 113(n=7)$ and $948 \pm 98 \mathrm{pA}(n=7)$, respectively $(p=0.36)$, rise times were $0.60 \pm 0.1$ and $0.58 \pm 0.07$ $\mathrm{ms}$, respectively $(p=0.82)$, and decay times were $1.0 \pm 0.12$ and $0.84 \pm 0.16 \mathrm{~ms}$, respectively $(p=0.46)$. 
Functional maturation of calyceal giant synapses in culture In patch-clamp whole-cell recordings from postsynaptic cells, fast synaptic currents could be evoked by extracellular stimulation of GFP-labeled input fibers after DIV15 (Fig. 2a). These synaptic currents were abolished by bath-applied CNQX $(25 \mu \mathrm{M})$, confirming that they were AMPA receptor-mediated, glutamatergic EPSCs. At DIV16-DIV18, the majority of postsynaptic cells innervated with calyceal terminals ( 8 of 14) received multiple inputs, judging from incremental steps of EPSC amplitude in response to gradually increased stimulus intensity (Fig. 2b). However, later at DIV20-DIV22, single calyceal innervations with all-or-none EPSC amplitudes in response to graded stimuli became predominant ( 12 of $13, p<0.01$, Mann-Whitney $U$ test). Thus, developmental elimination of calyceal and/or noncalyceal inputs likely occurs during culture. At cultured calyceal synapses before DIV13, input fiber stimulation failed to evoke EPSCs $(n=$ 9; Fig. 2c); but at DIV15, EPSCs were evoked at a majority of calyceal synapses (12 of 14 contacts) with a mean amplitude of $475 \pm 125 \mathrm{pA}(n=12)$. From DIV15 to DIV18, EPSCs became significantly larger $(878 \pm 75 \mathrm{pA}$ at DIV18, $p<0.05)$ and were evoked without exception (14 of 14). Mean amplitude of EPSCs did not increase further at DIV21. Concomitantly with the increased amplitude, rise and decay time kinetics of EPSCs became faster from DIV15 to DIV18 (Fig. 2c). At cultured calyceal synapses, spontaneous mEPSCs were rarely seen (DIV10-DIV21), as at calyces of Held a couple of days after synaptic formation (Chuhma and Ohmori, 1998) but could be revealed by increasing extracellular $\left[\mathrm{K}^{+}\right]$(from 2.5 to $25 \mathrm{~mm}$; Fig. $2 d$ ), even at DIV13 when EPSCs could not be evoked by fiber stimulation. The mean amplitude of mEPSCs was similar (32-38 pA) throughout DIV13-DIV21. Even in high extracellular $\left[\mathrm{K}^{+}\right]$solution, mEPSCs were undetectable at DIV10. The mEPSC frequency in the presence of $25 \mathrm{~mm}\left[\mathrm{~K}^{+}\right]$was relatively low at DIV13 $(\sim 2 \mathrm{~Hz})$, but tended to increase with culture days. Occurrence of $\left[\mathrm{K}^{+}\right]$induced mEPSCs at DIV13 when EPSCs cannot still be evoked by presynaptic action potential suggests that postsynaptic AMPA receptors are expressed before the presynaptic mechanism of synchronous neurotransmitter release is established. EPSCs evoked by a paired-pulse stimulation protocol showed a facilitation at DIV15, but a depression at DIV21, with the paired-pulse ratio (PPR; the second EPSC amplitude relative to the first one) decreasing during culture (Fig. 2e), suggesting a developmental increase in transmitter release probability during culture, as at the calyx of Held during the first postnatal week (Chuhma and Ohmori, 1998). In addition to these presynaptic developmental changes, postsynaptic development will also contribute to functional maturation of calyceal synapses (Soria Van Hoeve and Borst, 2010). Are structural changes correlated with functional changes in cultured calyceal synapses? We compared EPSCs recorded from postsynaptic cells innervated with calyceal terminals having early-stage (Stage 1 or 2) or late-stage (Stage 3 or 4 ) structures at each DIV $(15,18$, and 21$)$. With respect to the mean amplitude and rise time and decay time kinetics of EPSCs, there was no significant difference between synapses having early- or late-stage structures (Fig. $2 f$ ). Thus, in this culture system, structural and functional development of calyceal terminals seems to proceed independently.

\section{Imaging calyceal giant presynaptic terminals in culture}

Various imaging techniques were applicable to cultured calyceal terminals. Styryl dyes are widely used at peripheral and cultured synapses (Betz and Bewick, 1992) but rarely used in slices where background fluorescence from nonspecific bind- ing is high. Cultured calyceal terminals were loaded with the styryl dye, FM 4-64, and vesicle exocytosis triggered with 80 $\mathrm{mm} \mathrm{K}^{+}$was monitored as a fluorescent signal decline (Fig. $3 a$ ). The $\mathrm{pH}$-sensitive fluorescent marker, pHluorin, monitors intravesicular $\mathrm{pH}$ changes associated with vesicular endocytosis and exocytosis (Miesenböck et al., 1998). Transfection of synaptophysin-conjugated pHluorin (syp-pHluorin) in $\mathrm{CN}$ neurons stained resting calyceal terminals (Fig. 3b1). In calyceal terminals with high pHluorin expression, single action potential-induced pHluorin signals were recorded from part of a presynaptic terminal, simultaneously with EPSCs (Fig. 3b2). Single action potential-induced pHluorin signals were evident with a signal-to-noise ratio of $11.4 \pm 2.8(n=4)$ and a mean half-decay of $\sim 4 \mathrm{~s} \mathrm{(at} 37^{\circ} \mathrm{C}$; Fig. $3 b 3$ ). This decay time was similar regardless of the size of the calyx area (data not shown), suggesting little mixture of vesicles across the recording window during acquisition. The average number of vesicles exocytosed in the recording window can be estimated as $\sim 7$ from the EPSC amplitude (1.6 nA; 44 quanta with $36 \mathrm{pA}$ quantal size) and percentage of recording area versus whole terminal $(\sim 16 \%)$. As pHluorin expression on vesicles may not be $100 \%$, the number of vesicles giving rise to the pHluorin signal may be $<7$. Exocytosis of such a small number of vesicles in a fractional area of the calyx may underlie the size fluctuation of pHluorin signals despite constant EPSC amplitudes. Stronger stimulation with a repetitive pulse train $(50$ stimuli at $50 \mathrm{~Hz})$ produced a large signal $(\Delta \mathrm{F} / \mathrm{F}=153 \pm 6 \%)$ with a longer mean half-decay time of $10.2 \pm 0.6 \mathrm{~s}(n=$ 4; Fig. 3b4).

In contrast to $\mathrm{pHluorin}$, the $\mathrm{pH}$-sensitive fluorescent marker, CypHer, is bright at low $\mathrm{pH}$; therefore, it can be used for monitoring real-time vesicle dynamics in a presynaptic terminal. CypHer conjugated with synaptotagmin 2 antibody was loaded into vesicles through spontaneous endocytosis by overnight incubation in culture media. Within a resting calyceal terminal, spontaneous movements of CypHer-labeled vesicles were clearly observed in 3D directions in GFP-labeled calyceal terminals (Fig. $3 c$ ). Thus, this new preparation enables live imaging of vesicle movements within a giant presynaptic terminal.

\section{Direct whole-cell recording from calyceal presynaptic terminals in culture}

An important feature of giant presynaptic terminals is that their structure permits whole-cell pipette accessibility. Presynaptic $\mathrm{Ca}^{2+}$ currents (Fig. 4a) and membrane capacitance (Fig. $4 b$ ) were recorded from cultured calyceal terminals at physiological temperature (Fig. 4) as previously described for calyx of Held slice preparations (Sun and Wu, 2001; Yamashita et al., 2005). Furthermore, in this culture preparation, membrane capacitance and pHluorin signals can be simultaneously recorded (Fig. 4b2). When a train of stimulation (20 $\mathrm{ms} \times 20$ pulses at $20 \mathrm{~Hz}$ ) was given to a calyceal terminal, membrane capacitance underwent an exocytic increase followed by an endocytic decline. Simultaneously monitored pHluorin signals underwent an increase in parallel with the membrane capacitance but decayed more slowly than the membrane capacitance, with a significant difference in the remaining signal at $50 \mathrm{~s}$ after the end of the stimulation train $(p<0.05)$. Because the decay phase of the pHluorin signal includes both endocytic and vesicle acidification times (Atluri and Ryan, 2006), the slower decay of the pHluorin signal suggests a membrane compartment undergoing slow acidification following endocytosis. 
a

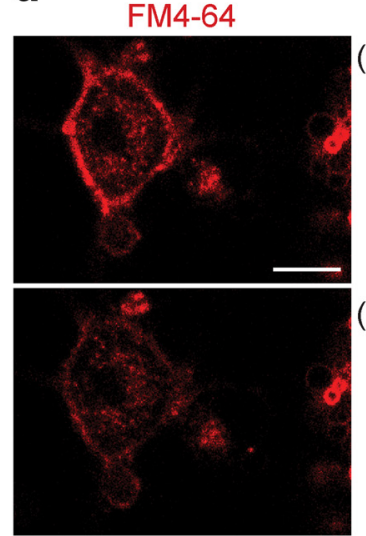

(i)

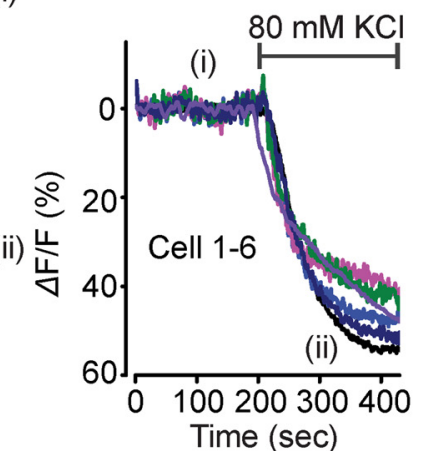

C
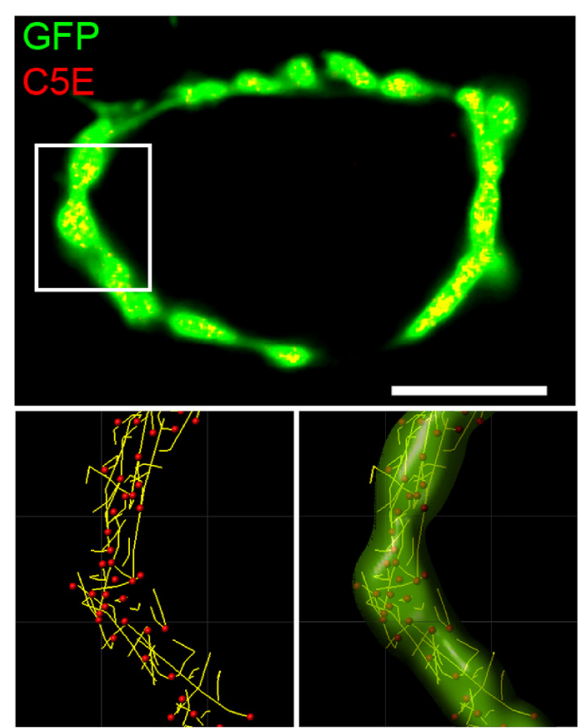

b1
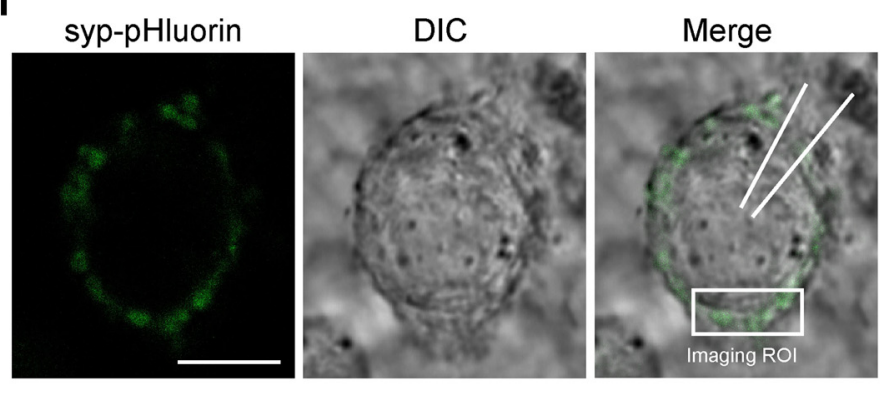

b2
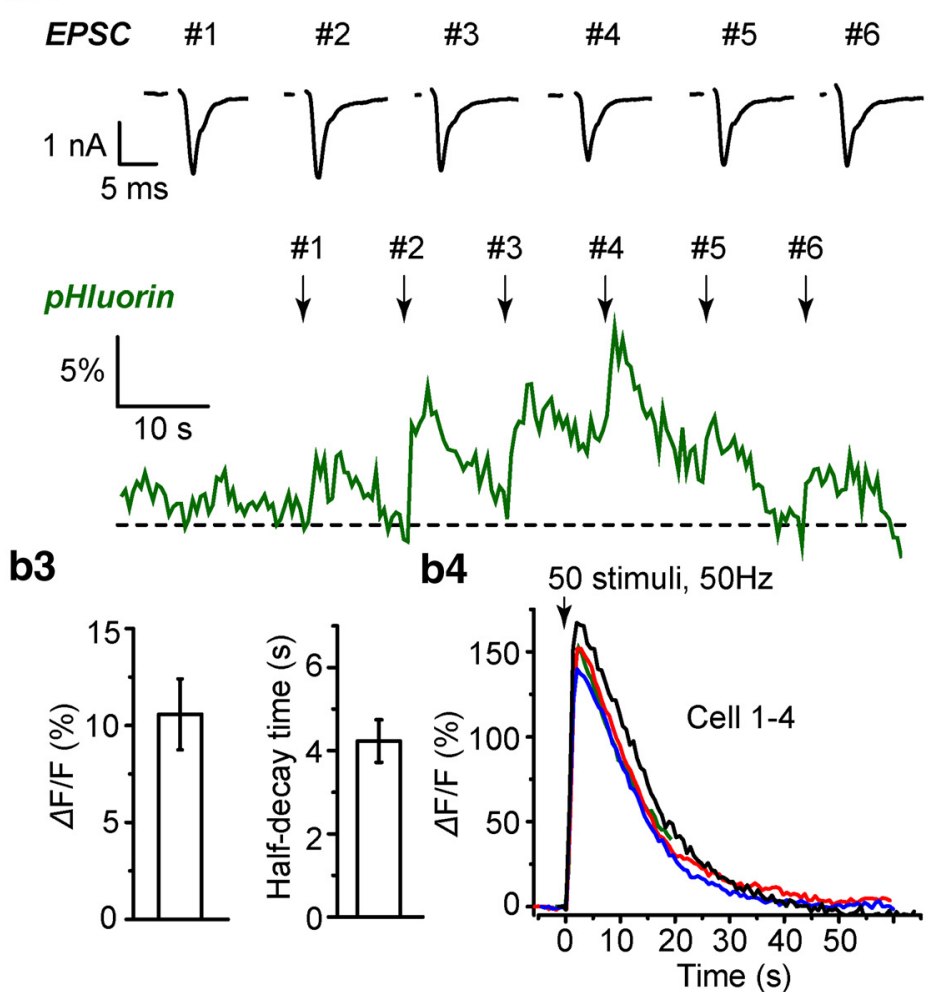

Figure 3. Imaging of synaptic vesicle dynamics. $\boldsymbol{a}$, Calyceal terminal loaded with FM4-64 before (top) and after (bottom) application of 80 mm KCl. Right, Destaining time courses of terminal-loaded FM dye (6 calyceal terminals, superimposed) at DIV19. Half-decay time was $63.4 \pm 12$ s. b1, A calyceal terminal in culture (DIV19) expressing syp-pHluorin. Scale bar, $10 \mu$ m. Resting syp-pHluorin fluorescence (left), DIC (middle), and merged (right) images of calyceal terminals used for simultaneous patch-clamp recording and imaging of exo-endocytosis in a single synaptic terminal. The postsynaptic patch pipette and imaging area (ROI) are illustrated. $\boldsymbol{b} 2$, Simultaneous recording of EPSCs (top row) and pHluorin signal (bottom row, green) evoked by 6 single stimuli at $0.1 \mathrm{~Hz}$ in a calyceal terminal. $\boldsymbol{b} 3$, Mean pHluorin signal amplitude ( $\Delta \mathrm{F} / \mathrm{F}$, left) and mean half-decay time (right, $n=4$ calyces) evoked by a single stimulus. $\boldsymbol{b} 4$, PHluorin fluorescence signals from 4 calyces (superimposed) evoked by stimulation at $50 \mathrm{~Hz}$ for $1 \mathrm{~s}$. c, Live imaging of synaptic vesicle dynamics in a GFP-labeled cultured calyceal terminal using synaptotagmin 2 (luminal) antibody-conjugated CypHer dye. Top, Cytosolic GFP (green) and CypHer (red) fluorescence signals after CypHer loading. Scale bar, $5 \mu \mathrm{m}$. Bottom left, Trajectories (yellow) of CypHer-loaded vesicles tracking within the area indicated by a square in the top picture (red) over 30 s period. Bottom right, 3D rendering of the terminal area superimposed with vesicle trajectories.

shRNA knockdown of presynaptic proteins in calyceal terminals

For genetic manipulation of cultured giant synapse preparations, we tested shRNA knockdown of a presynaptic vesicular protein. Among vesicle glutamate transporters, both VGLUT1 and VGLUT2 are expressed in cultured calyceal terminals (Fig. 1e) as in situ calyces of Held (Billups, 2005; Blaesse et al., 2005). VGLUT1 knock-out mice survive postnatal weeks (Fremeau et al., 2004; Wojcik et al., 2004), but VGLUT2 knock-out mice undergo perinatal lethality (Moechars et al., 2006); therefore, the culture system is required for examining their phenotypes. We knocked down VGLUT2 in culture using lentivirus-mediated shRNA. Ten days after lentiviral infection, VGLUT2 expression, as deduced from immunofluorescence signal intensity, was significantly reduced $(p<0.05)$, whereas VGLUT1 expression re- mained unchanged (Fig. 5a). In the preparation with VGLUT2 knockdown, evoked EPSCs were significantly smaller in amplitude than controls infected with scrambled shRNA $(p<0.05$; Fig. 5b). However, there was no significant difference in the PPR or the coefficient of variation of EPSC amplitude, suggesting that VGLUT2 knockdown reduced the vesicular glutamate content without affecting the release probability or the number of readily releasable vesicles. These results are consistent with the report of reduced miniature EPSC amplitude at cultured thalamic synapses in VGLUT2 knock-out mice (Moechars et al., 2006). However, unchanged PPR after VGLUT2 knockdown is incompatible with the hypothesis proposed in hippocampal culture that the release probability of VGLUT2 vesicles is higher than that of VGLUT1 vesicles (Weston et al., 2011). Thus, the loss-offunction studies using shRNA knockdown in cultured calyceal 
a1

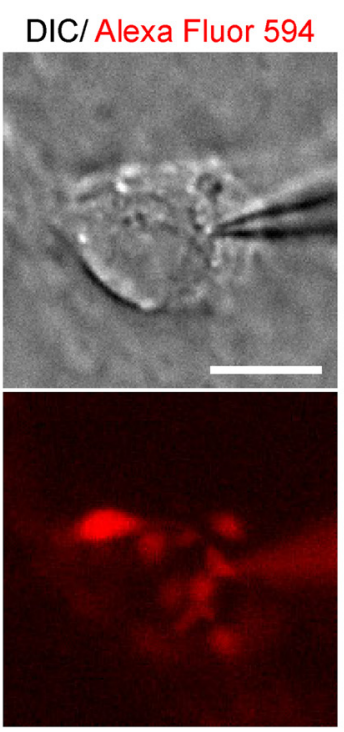

a2

a3
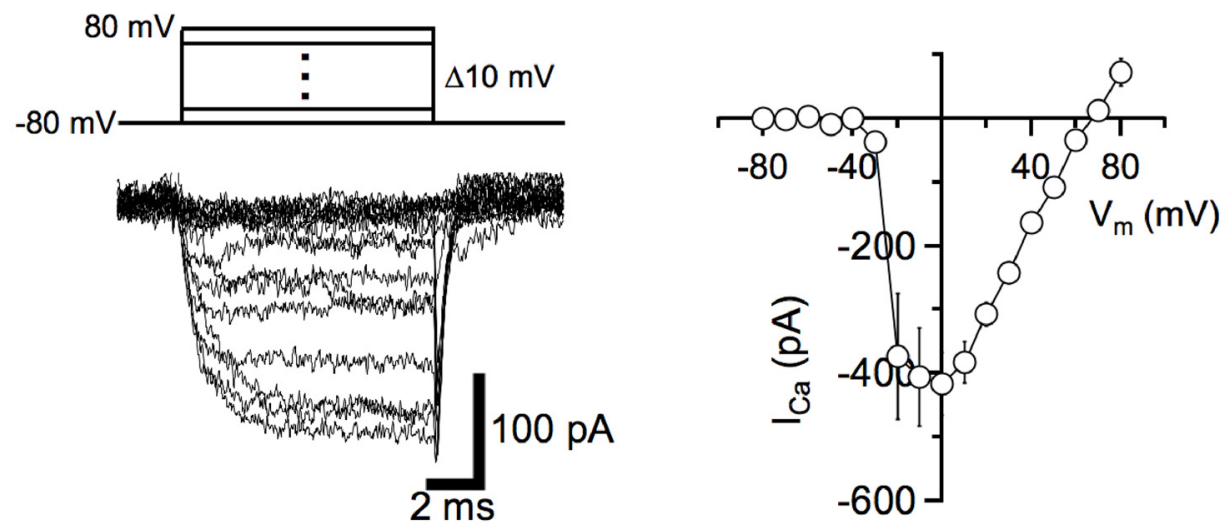

b1

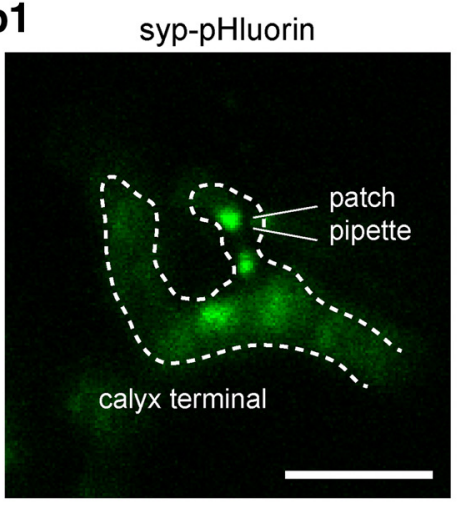

b2

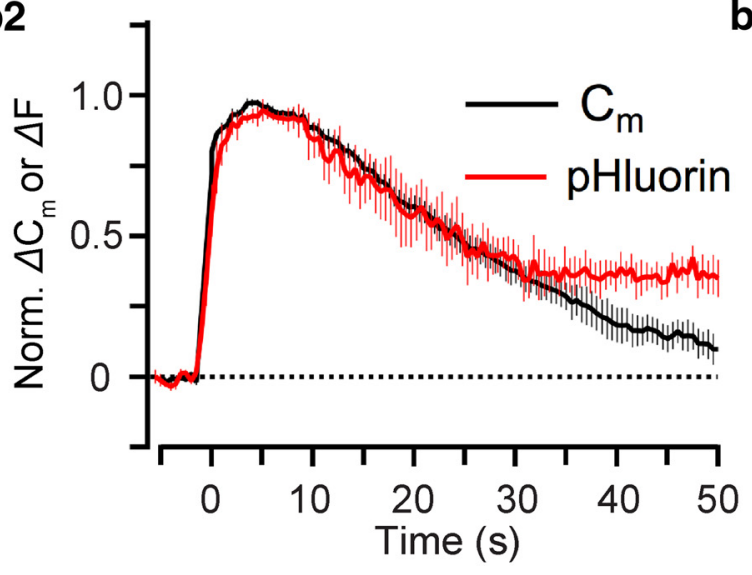

b3

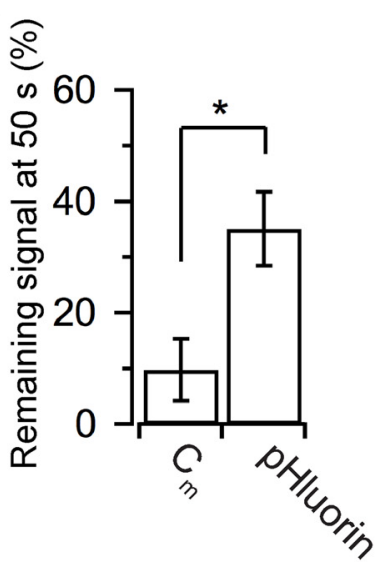

Figure 4. Whole-cell patch-clamp recordings from cultured calyceal terminals. $\boldsymbol{a}$, Whole-cell patch-clamp recording of $\mathrm{Ca}^{2+}$ currents from cultured calyceal terminals. $\boldsymbol{a}$ 1, Top, DIC image of a cultured calyceal terminal with a patch pipette. Bottom, Calyceal terminal injected with AlexaFluor-594 (red) via the patch pipette. Scale bar, $10 \mu \mathrm{m} . \mathbf{a}_{2}$, Sample traces of voltage-gated Ca ${ }^{2+}$ channel currents (superimposed) recorded from a cultured calyceal terminal. Ca ${ }^{2+}$ currents were evoked with square pulses $(10 \mathrm{~ms})$ stepping, by $10 \mathrm{mV}$, from the holding potential $(-80 \mathrm{mV})$ to $80 \mathrm{mV}$ (voltage command protocol on the top). $\boldsymbol{a}$ 3, The current-voltage relationship of $\mathrm{Ca}^{2+}$ channel currents obtained from 6 presynaptic terminals. $\boldsymbol{b}$, Simultaneous recording of membrane capacitance changes and pHluorin signals from calyceal terminals in culture. $\boldsymbol{b} 1$, Resting syp-pHluorin epifluorescence image of a cultured calyceal terminal (green). Dotted line indicates terminal contour. The patch pipette position is indicated by a drawing. Scale bar, $10 \mu \mathrm{m}$. $\boldsymbol{b 2}$, Simultaneously recorded membrane capacitance change $\left(\mathrm{C}_{\mathrm{m}}\right.$, black trace) and pHluorin signal $(\Delta \mathrm{F}$, red trace), which were evoked by a train of repetitive stimulations ( $20 \mathrm{~ms}$ square pulses from -80 to $10 \mathrm{mV}, 20$ times at $20 \mathrm{~Hz}$ ). Data from 5 terminals were averaged and aligned at the onset of stimulation. Peak amplitudes were scaled at the peaks. $\boldsymbol{b 3}$, Percentages of membrane capacitance and pHluorin fluorescence remaining 50 s after the train of stimulation; $n=5 .{ }^{*} p<0.05 . \boldsymbol{a}, \boldsymbol{b}$, Recordings were made at DIV18-DIV20.

synapses can provide answers to various biological questions about presynaptic molecules.

\section{Discussion}

We have developed a giant synapse preparation in dissociated cell culture. In this preparation, it is possible to perform whole-cell recording from presynaptic terminals simultaneously with optical imaging (Fig. 4). This enabled dissecting pHluorin signals into vesicle acidification time and vesicle endocytic time (Fig. 4b). By extending this advantage, it will also be possible to monitor presynaptic molecular interactions using optical imaging methods (e.g., fluorescence resonance energy transfer) while monitoring exo-endocytosis using membrane capacitance measurements. As presynaptic whole-cell recording (Fig. 4) and shRNA-KD techniques (Fig. 5) work well in the newly developed cultured giant presynaptic terminal, exocytic, endocytic, or the $\mathrm{Ca}^{2+}$ channel- modulating role of presynaptic molecules can accurately be determined.

Overexpression of synaptic proteins or their mutants in the calyx of Held, using in vivo stereotaxic viral transfer techniques, revealed various roles of presynaptic proteins (Wimmer et al., 2004; Young and Neher, 2009; Schwenger and Kuner, 2010). Compared with the aforementioned technique, cultured calyces are technically easier to manipulate and offer broader applications, particularly with shRNA knockdown, that have not yet been successfully used with in vivo viral transfer techniques (Wimmer et al., 2004; Schneggenburger and Forsythe, 2006). Cultured calyceal presynaptic terminals are also ideal for imaging synaptic organelles. The large structure of cultured calyceal terminals enables live monitoring of $3 \mathrm{D}$ vesicle dynamics along long tracks $(<20 \mu \mathrm{m})$, whereas vesicle imaging at bouton-like synapses is highly restricted in space. With the feasibility of genetic 
a
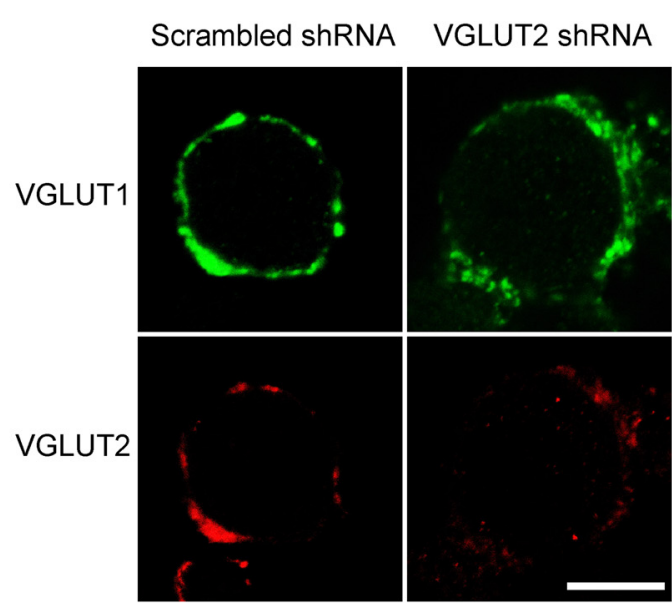

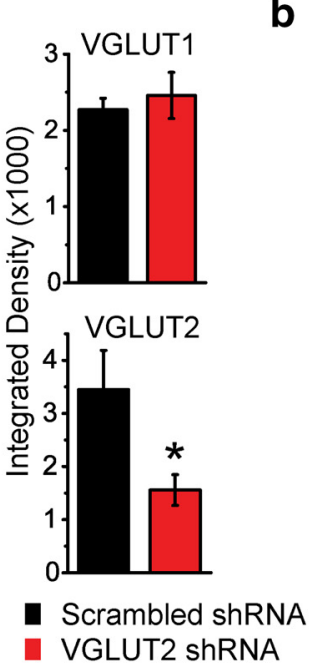

b

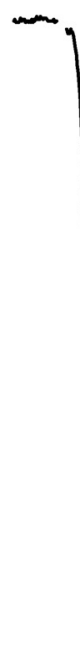

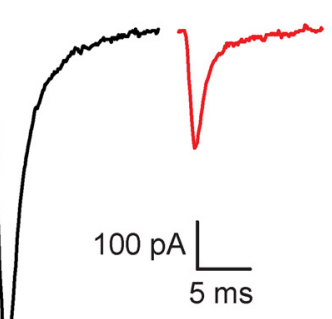
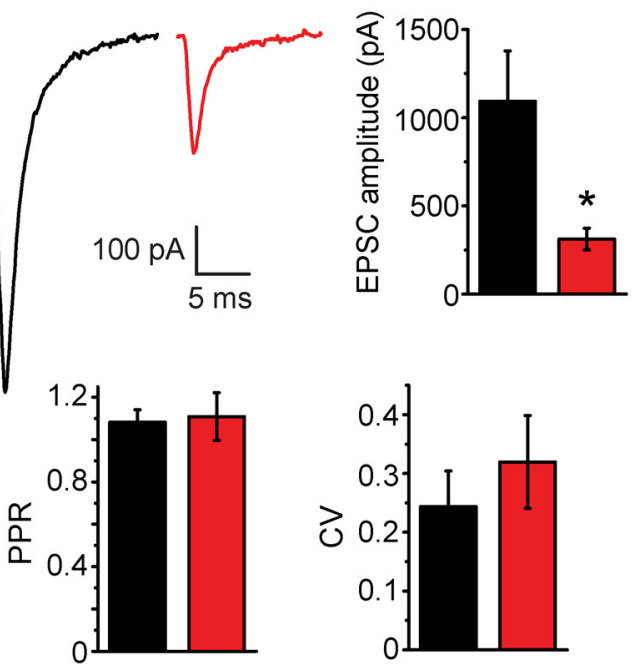

Figure 5. shRNA knockdown of presynaptic proteins in calyceal terminals in culture. Cultured cells were infected at DIV8 with shRNA lentiviral particles specific for VGLUT2. Scrambled shRNA lentiviral particles were used as a negative control. Experiments were performed at DIV18. $\boldsymbol{a}$, VGLUT1 (top panels; green) and VGLUT2 (bottom panels; red) immunofluorescence images of calyceal terminals infected with either scrambled shRNA (left panels) or VGLUT2-specific shRNA (right panels). Scale bar, $10 \mu \mathrm{m}$. Bar graphs represent immunofluorescence signal intensities of VGLUT1 (top) and VGLUT2 (bottom) after infection with scrambled (black bars) orVGLUT 2-specific (red bars) shRNAs. Apparent knockdown efficiency was $55 \%$ for VGLUT2 ( $n=11$ each, $p=0.027$ compared with scrambled shRNA; for details, see Materials and Methods). $\boldsymbol{b}$, Evoked EPS(s at calyceal synapses in culture infected with scrambled (black) or VGLUT2-specific (red) shRNAs. Bar graphs represent mean EPSC amplitudes (1092 $\pm 286 \mathrm{pA}$ in scrambled shRNA control vs $311 \pm 61 \mathrm{pA}$ in VGLUT2-shRNA; $n=5$ and $n=6, p=0.017$ ). Bottom left, Bar graphs represent the PPR at interpulse intervals of $20 \mathrm{~ms}$. Bottom right, Bar graphs represent the coefficient of variation ( $C V=S D /$ mean) of the EPSC amplitude. Bar graphs are color-coded as EPSC traces. ${ }^{*} p<0.05$.

manipulation and intraterminal access, this preparation offers diverse opportunities to study basic molecular mechanisms underlying presynaptic functions, synaptogenesis, synaptic maturation, and vesicle dynamics.

In addition to bouton-like small synapses, there are many large synapses in the CNS, such as mossy fiber pyramidal cell synapses in the hippocampus, mossy fiber granule cell synapses, basket cell Purkinje cell synapses, climbing fiber Purkinje cell synapses, and Purkinje cell deep nuclear cell synapses in the cerebellum, and many auditory relay synapses in the brainstem, including the calyx of Held and endbulb of Held, playing detonator roles in neurotransmission (Rancz et al., 2007). Giant synapses established in this culture system raise the question of whether there is a specific molecular mechanism underlying large synapse formation. In our culture conditions, supplemented with astrocyte-conditioned medium, NT3 was required for efficient calyx formation, whereas BDNF was dispensable, suggesting that the NT3 high-affinity receptor TrkC plays an important role in giant synapse formation. In a hippocampal neuron-fibroblast coculture system, it has been reported that the leucine-rich repeat region of TrkC is essential for upregulation of presynaptic and postsynaptic molecules (Takahashi et al., 2011). The lack of structure-function correlation in developing calyceal synapses in culture is in line with distinct molecular cascades downstream of NT3/TrkC reportedly modifying structural or functional changes at cultured neuromuscular synapses (Je et al., 2006). Genetic manipulations of TrkC and its downstream molecules might reveal a specific molecular cascade involved in giant synapse formation. In addition to NT3/TrkC, factors, such as Wnts (Cerpa et al., 2008; Dickins and Salinas, 2013), Ephrin (Lim et al., 2008; Hsieh et al., 2010), and/or BMP (Xiao et al., 2013), may participate in calyceal synaptic maturation in situ. Testing different molecular cues in this calyceal giant synapse culture model would provide hints as to the molecules involved in development of giant synapses in situ; thus, they can be used to address molecular mechanisms underlying synapse size and giant synapse formation and maturation.
The present culture preparation contains cells other than globular bushy cells and MNTB principal neurons. We labeled CN cells with GFP before coculture to ensure that GFPpositive terminals were derived from $\mathrm{CN}$ cells. In chick embryo dissociated cell culture, Edinger-Westphal neurons form calyceal contacts specifically on ciliary ganglion neurons (Fujii and Berg, 1987), and calyceal contact is rare in EdingerWestphal alone cultures (Fujii and Berg, 1987; Fujii, 1994). Similarly, calyceal synapses were formed much less frequently in $\mathrm{CN}$ alone cultures (2/dish) than in CN-MNTB cocultures (13/dish). Thus, the majority of calyceal synapses are likely formed between cells in CN and MNTB regions in our culture system. Specific labeling of both globular bushy cells and MNTB principal neurons would be required in the future to distinguish the calyx of Held synapse from other calyx-type synapses in culture.

With respect to structural development, cultured calyceal terminals share some similarities with calyces of Held (Wimmer et al., 2006), such as finger-like processes containing many swelling at later stages of development. However, unlike development of calyces of Held in situ, the spoon-shaped structure and subsequent fenestration (Kandler and Friauf, 1993) were not observed during calyceal synaptic maturation in culture. Developmental synaptic elimination occurs in culture, like developing calyces of Held (Hoffpauir et al., 2006; Holcomb et al., 2013), but competition between calyceal terminals (Hoffpauir et al., 2006; Holcomb et al., 2013) was not evident in culture. The PPR of EPSCs in cultured calyceal synapses decreased from DIV15 to DIV21, suggesting a developmental increase in transmitter release probability. At calyces of Held of developing rodents, transmitter release probability increases during the first postnatal week (Chuhma and Ohmori, 1998) but decreases during the second postnatal week (Koike-Tani et al., 2008). Together with small EPSC amplitude and rare occurrences of spontaneous EPSCs, cultured calyceal synapses at DIV18-DIV21 are comparable with calyces of Held of P3-P4 rodents (Chuhma and Ohmori, 1998; 
Hoffpauir et al., 2006). It remains to be determined which factors promote maturation of cultured calyceal synapses beyond this developmental stage.

\section{References}

Allen Developing Mouse Brain Atlas. http://developingmouse.brain-map.org.

Allen NJ, Bennett ML, Foo LC, Wang GX, Chakraborty C, Smith SJ, Barres BA (2012) Astrocyte glypicans 4 and 6 promote formation of excitatory synapses via GluA1 AMPA receptors. Nature 486:410-414. CrossRef Medline

Atluri PP, Ryan TA (2006) The kinetics of synaptic vesicle reacidification at hippocampal nerve terminals. J Neurosci 26:2313-2320. CrossRef Medline

Betz WJ, Bewick GS (1992) Optical analysis of synaptic vesicle recycling at the frog neuromuscular junction. Science 10:200-203. CrossRef Medline

Billups B (2005) Colocalization of vesicular glutamate transporters in the rat superior olivary complex. Neurosci Lett 382:66-70. CrossRef Medline

Blaesse P, Ehrhardt S, Friauf E, Nothwang HG (2005) Developmental pattern of three vesicular glutamate transporters in the rat superior olivary complex. Cell Tissue Res 320:33-50. CrossRef Medline

Borst JG, Helmchen F, Sakmann B (1995) Pre- and postsynaptic whole-cell recordings in the medial nucleus of the trapezoid body of the rat. J Physiol 489:825-840. CrossRef Medline

Cerpa W, Godoy JA, Alfaro I, Farías GG, Metcalfe MJ, Fuentealba R, Bonansco C, Inestrosa NC (2008) Wnt-7a modulates the synaptic vesicle cycle and synaptic transmission in hippocampal neurons. J Biol Chem 283:5918-5927. CrossRef Medline

Chuhma N, Ohmori H (1998) Postnatal development of phase-locked high-fidelity synaptic transmission in the medial nucleus of the trapezoid body of the rat. J Neurosci 18:512-520. Medline

De Lorenzo AJ (1960) The fine structure of synapses in the ciliary ganglion of the chick. J Biophys Biochem Cytol 7:31-36. CrossRef Medline

Dickins EM, Salinas PC (2013) Wnts in action: from synapse formation to synaptic maintenance. Front Cell Neurosci 7:162. CrossRef Medline

Eguchi K, Nakanishi S, Takagi H, Taoufiq Z, Takahashi T (2012) Maturation of a PKG-dependent retrograde mechanism for exoendocytic coupling of synaptic vesicles. Neuron 74:517-529. CrossRef Medline

Forsythe ID (1994) Direct patch recording from identified presynaptic terminals mediating glutamatergic EPSCs in the rat CNS, in vitro. J Physiol 479:381-387. CrossRef Medline

Fremeau RT Jr, Kam K, Qureshi T, Johnson J, Copenhagen DR, StormMathisen J, Chaudhry FA, Nicoll RA, Edwards RH (2004) Vesicular glutamate transporters 1 and 2 target to functionally distinct synaptic release sites. Science 304:1815-1819. CrossRef Medline

Fujii JT (1994) Synaptic vesicle proteins and target-dependent morphogenesis of calyx-like contacts in vitro. Exp Neurol 129:155-162. CrossRef Medline

Fujii JT, Berg DK (1987) Formation of calyx-like contacts preferentially on appropriate target neurons in culture. Dev Biol 123:346-353. CrossRef Medline

Gaffield MA, Betz WJ (2006) Imaging synaptic vesicle exocytosis and endocytosis with FM dyes. Nat Protocols 1:2916-2921. CrossRef Medline

Hoffpauir BK, Grimes JL, Mathers PH, Spirou GA (2006) Synaptogenesis of the calyx of Held: rapid onset of function and one-to-one morphological innervation. J Neurosci 26:5511-5523. CrossRef Medline

Holcomb PS, Hoffpauir BK, Hoyson MC, Jackson DR, Deerinck TJ, Marrs GS, Dehoff M, Wu J, Ellisman MH, Spirou GA (2013) Synaptic inputs compete during rapid formation of the calyx of Held: a new model system for neural development. J Neurosci 33:12954-12969. CrossRef Medline

Hsieh CY, Nakamura PA, Luk SO, Miko IJ, Henkemeyer M, Cramer KS (2010) Ephrin-B reverse signaling is required for formation of strictly contralateral auditory brainstem pathways. J Neurosci 30:9840-9849. CrossRef Medline

Je HS, Yang F, Zhou J, Lu B (2006) Neurotrophin 3 induces structural and functional modification of synapses through distinct molecular mechanisms. J Cell Biol 175:1029-1042. CrossRef Medline

Kandler K, Friauf E (1993) Pre- and postnatal development of efferent connections of the cochlear nucleus in the rat. J Comp Neurol 328:161-184. CrossRef Medline

Koike-Tani M, Kanda T, Saitoh N, Yamashita T, Takahashi T (2008) Involvement of AMPA receptor desensitization in short-term synaptic depression at the calyx of Held in developing rats. J Physiol 586:2263-2275. CrossRef Medline

Lim BK, Matsuda N, Poo MM (2008) Ephrin-B reverse signaling promotes structural and functional synaptic maturation in vivo. Nat Neurosci 11: 160-169. CrossRef Medline
Lohmann C, Ilic V, Friauf E (1998) Development of a topographically organized auditory network in slice culture is calcium dependent. J Neurobiol 34:97-112. CrossRef Medline

Marrs GS, Morgan WJ, Howell DM, Spirou GA, Mathers PH (2013) Embryonic origins of the mouse superior olivary complex. Dev Neurobiol 73:384-398. CrossRef Medline

Matsuda T, Cepko CL (2004) Electroporation and RNA interference in the rodent retina in vivo and in vitro. Proc Natl Acad Sci U S A 101:16-22. CrossRef Medline

Miesenböck G, De Angelis DA, Rothman JE (1998) Visualizing secretion and synaptic transmission with $\mathrm{pH}$-sensitive green fluorescent proteins. Nature 394:192-195. CrossRef Medline

Moechars D, Weston MC, Leo S, Callaerts-Vegh Z, Goris I, Daneels G, Buist A, Cik M, van der Spek P, Kass S, Meert T, D’Hooge R, Rosenmund C, Hampson RM (2006) Vesicular glutamate transporter VGLUT2 expression levels control quantal size and neuropathic pain. J Neurosci 26: 12055-12066. CrossRef Medline

Ramon y Cajal S (1911) Histologie du système nerveux de l'homme et des vertébrés. Paris: Maloine.

Rancz EA, Ishikawa T, Duguid I, Chadderton P, Mahon S, Häusser M (2007) High-fidelity transmission of sensory information by single cerebellar mossy fibre botons. Nature 450:1245-1248. CrossRef Medline

Schneggenburger R, Forsythe ID (2006) The calyx of Held. Cell Tissue Res 326:311-337. CrossRef Medline

Schwenger DB, Kuner T (2010) Acute genetic perturbation of exocyst function in the rat calyx of Held impedes structural maturation, but spares synaptic transmission. Eur J Neurosci 32:974-984. CrossRef Medline

Soria Van Hoeve JS, Borst JG (2010) Delayed appearance of the scaffolding proteins PSD-95 and Homer-1 at the developing rat calyx of Held synapse. J Comp Neurol 518:4581-4590. CrossRef Medline

Sun JY, Wu LG (2001) Fast kinetics of exocytosis revealed by simultaneous measurements of presynaptic capacitance and postsynaptic currents at a central synapse. Neuron 30:171-182. CrossRef Medline

Takahashi H, Arstikaitis P, Prasad T, Bartlett TE, Wang YT, Murphy TH, Craig AM (2011) Postsynaptic TrkC and presynaptic PTP $\sigma$ function as a bidirectional excitatory synaptic organizing complex. Neuron 69: 287-303. CrossRef Medline

Takahashi T, Forsythe ID, Tsujimoto T, Barnes-Davies M, Onodera K (1996) Presynaptic calcium current modulation by a metabotropic glutamate receptor. Science 274:594-597. CrossRef Medline

Tong H, Steinert JR, Robinson SW, Chernova T, Read DJ, Oliver DL, Forsythe ID (2010) Regulation of Kv channel expression and neuronal excitability in rat medial nucleus of the trapezoid body maintained in organotypic culture. J Physiol 588:1451-1468. CrossRef Medline

Wellsall J (1956) Studies on the structure and innervation of the sensory epithelium of the cristae ampulares in the guinea pig: a light and electron microscopic investigation. Acta Otolaryngol Suppl 126:1-85. Medline

Weston MC, Nehring RB, Wojcik SM, Rosenmund C (2011) Interplay between VGLUT isoforms and endophilin Al regulates neurotransmitter release and short-term plasticity. Neuron 69:1147-1159. CrossRef Medline

Wimmer VC, Nevian T, Kuner T (2004) Targeted in vivo expression of proteins in the calyx of Held. Pflugers Arch 449:319-333. CrossRef Medline

Wimmer VC, Horstmann H, Groh A, Kuner T (2006) Donut-like topology of synaptic vesicles with a central cluster of mitochondria wrapped into membrane protrusions: a novel structure-function module of the adult calyx of Held. J Neurosci 26:109-116. CrossRef Medline

Wojcik SM, Rhee JS, Herzog E, Sigler A, Jahn R, Takamori S, Brose N, Rosenmund C (2004) An essential role for vesicular glutamate transporter 1 (VGLUT1) in postnatal development and control of quantal size. Proc Natl Acad Sci U S A 101:7158-7163. CrossRef Medline

Xiao L, Michalski N, Kronander E, Gjoni E, Genoud C, Knott G, Schneggenburger R (2013) BMP signaling specifies the development of a large and fast CNS synapse. Nat Neurosci 16:856-864. CrossRef Medline

Yamashita T, Hige T, Takahashi T (2005) Vesicle endocytosis requires dynamin-dependent GTP hydrolysis at a fast CNS synapse. Science 307: 124-127. CrossRef Medline

Young SM Jr, Neher E (2009) Synaptotagmin has an essential function in synaptic vesicle positioning for synchronous release in addition to its role as a calcium sensor. Neuron 63:482-496. CrossRef Medline

Zhu Y, Xu J, Heinemann SF (2009) Two pathways of synaptic vesicle retrieval revealed by single-vesicle imaging. Neuron 61:397-411. CrossRef Medline 